\title{
Corela
}

Cognition, représentation, langage

HS-30 | 2020

Phonétique, littérature et enseignement du FLE : théories et recherches

\section{Adopter des techniques d'acteur pour interpréter un texte en classe de langue}

\section{François Blondel}

\section{(2) OpenEdition}

\section{Journals}

Édition électronique

URL : http://journals.openedition.org/corela/10286

DOI : 10.4000/corela. 10286

ISSN : 1638-573X

Éditeur

Cercle linguistique du Centre et de I'Ouest - CerLICO

Référence électronique

François Blondel, «Adopter des techniques d'acteur pour interpréter un texte en classe de langue », Corela [En ligne], HS-30 | 2020, mis en ligne le 30 avril 2020, consulté le 22 mai 2020. URL : http:// journals.openedition.org/corela/10286 ; DOI : https://doi.org/10.4000/corela.10286

Ce document a été généré automatiquement le 22 mai 2020.

\section{(c) (i) (9)}

Corela - cognition, représentation, langage est mis à disposition selon les termes de la licence Creative Commons Attribution - Pas d'Utilisation Commerciale - Partage dans les Mêmes Conditions 4.0 International. 


\title{
Adopter des techniques d'acteur pour interpréter un texte en classe de langue
}

\author{
François Blondel
}

«J'aime beaucoup les exercices de lecture. [...] En littérature, les phrases obéissent à un rythme, tout comme en musique. Le comédien doit repérer la composition rythmique de la phrase. [...]. Personnellement, j'aime me tenir au plus proche de l'auteur et le faire entendre lui. Pas

question de chanter ma musique à moi. »

(Renucci, 2014 : 156-158)

1 L'interprétation des textes dramatiques ou poétiques figure parmi les outils d'enseignement de la prononciation d'une langue. L'apprenant n'a certes pas vocation à être acteur, mais interpréter un texte dans la langue cible le motive. L'objectif des activités d'interprétation n'est pas d'incarner un personnage, ni de toucher un public, mais de lui permettre de se libérer des automatismes acquis dans sa propre langue. Pour ce faire, il a d'abord recours à l'imitation, grâce aux enregistrements qui accompagnent les livres et manuels, mais il s'agit pour lui aussi de savoir déchiffrer les graphèmes, d'identifier les liaisons et les enchaînements, de respecter le découpage rythmique. Comment alors s'approprier un texte écrit, pour le dire de façon naturelle et spontanée, sans se limiter à la récitation dans ces conditions?

2 Les acteurs, pour éviter les risques de créer des mécanismes lors des répétitions, font appel à différentes techniques vocales, corporelles ou d'improvisation, qui non seulement facilitent la mémorisation, mais aussi permettent d'acquérir une certaine disponibilité lors de l'interprétation, pour varier leur jeu, oublier leur technique, afin d'atteindre le naturel recherché.

3 Il semble pertinent d'emprunter certaines de ces techniques en classe de langue, pour permettre aux apprenants de puiser en eux-mêmes tout ce qui leur permet d'atteindre 
une prononciation naturelle, de varier leurs intonations, tout en prenant conscience de leurs erreurs, de les corriger, grâce à l'acquisition progressive d'une souplesse articulatoire, à une disponibilité physique et à une amélioration de leur capacité d'écoute.

Dans cette contribution, nous présentons différents exercices conçus pour des acteurs, adaptables en classe de langue, dont l'objectif est de travailler, lors de l'interprétation d'un texte, la mobilité des organes de l'articulation, la relation entre le corps et le texte, et la conscience de l'instant présent lors de son interprétation. Ces exercices ont été testés lors de plusieurs stages de formation dont un de huit heures, de phonétique et d'expression orale en FLE, à l'ENS de Lyon en 2015, auprès d'apprenants du CHELS, de différentes nationalités, du niveau B1 au C2. Dans ces stages, la majorité des apprenants prennent conscience de leurs erreurs de prononciation après avoir corrigé ponctuellement l'articulation des phonèmes qui leur posaient problème et acquièrent une intonation plus naturelle lors de la lecture à voix haute des textes supports. La plupart de ces exercices peuvent s'adapter à tout type de texte à oraliser (théâtre en vers, en prose, poésie, fables, chansons...). Nous nous concentrons sur la phase d'interprétation d'un texte, qui suit souvent tout un travail de discrimination auditive et d'entraînement. Le texte support est choisi en fonction du niveau des apprenants et des objectifs de l'enseignant. Il s'agit de proposer ici des alternatives pour exploiter une scène, une page ou un poème, cette approche du texte étant nécessairement partielle. Les 500 exercices de phonétique (Abry et Chalaron, 2010 pour les niveaux A1/A2, et 2011 pour les niveaux B1/B2) offrent de très nombreux extraits littéraires dans lesquels peuvent puiser les enseignants.

\section{Déchiffrer le texte et le « mettre en voix »}

5 Les textes de théâtre écrits en prose peuvent sembler faciles à aborder, car ils sont écrits pour être oralisés. On identifie rapidement les locuteurs et la longueur des répliques. Certains textes vont même jusqu'à indiquer les pauses et les silences. Cette simplicité apparente peut constituer un piège, dans la mesure où on est tenté, dès les premières lectures, de jouer les dialogues, en reproduisant les intonations les plus évidentes, codées selon nos représentations de la comédie, de la tragédie, du mélodrame, ou de toute marque d'expressivité. Il convient donc de découvrir le texte de la façon la plus neutre possible, afin de ne pas lui imposer une interprétation trop évidente, et de ne pas créer d'automatismes.

6 Comme l'acteur qui effectue ses premières lectures sans la moindre intention de jeu, chaque apprenant découvre le texte comme une matière brute pour mieux en sentir les nuances de sens. Cette phase de déchiffrage est d'autant plus primordiale chez l'apprenant de langue étrangère, qu'il s'agit pour lui de différencier le code écrit du code oral, de respecter un découpage rythmique et de prononcer des sons qui ne lui sont pas naturels. Ce qui est considéré comme une lecture à plat chez l'acteur consiste chez l'apprenant en un véritable exercice d'entraînement articulatoire et prosodique. Afin de faciliter son travail, il est possible de l'inviter à "mettre en bouche le texte", pour prendre conscience du rôle des différents organes de la parole. 


\section{1. « Mettre en bouche » le texte}

7 En guise de déchiffrage vocal, le metteur en scène Michel Liard, proposait à ses acteurs et ses élèves, un exercice d'incorporation du texte par les phonèmes. Il comparait le texte à une matière à façonner, dont il fallait sculpter les sons. Plus qu'un simple exercice de diction, il s'agissait de sensibiliser l'élève-acteur à toute la musicalité du texte, recherchée par l'auteur, aux allitérations, au choix des sons, dès la première approche. Cet exercice, permet à l'apprenant ${ }^{1}$ d'éviter certaines erreurs de lecture des graphèmes, de respecter les liaisons et les enchaînements.

Ce déchiffrage consiste à séparer dans un premier temps les consonnes et les voyelles, en remplaçant toutes les voyelles par une voyelle unique ou en n'articulant que les voyelles. Un exemple avec cet extrait de Roberto Zucco de B.-M. Koltès (1990 : 23-24) :

LA GAMINE. - Enlève tes chaussures. Comment t'appelles-tu?

ZUCCO. - Appelle-moi comme tu veux. Et toi?

LA GAMINE. - Moi, je n'ai plus de nom. [...]. Qu'est-ce que tu fais, dans la vie?

ZUCCO. - Dans la vie?

LA GAMINE. - Oui, dans la vie : ton métier, ton occupation, comment tu gagnes de

l'argent, et toutes ces choses que tout le monde fait?

ZUCCO. - Je ne fais pas ce que fait tout le monde.

LA GAMINE. - Alors justement, dis-moi ce que tu fais.

9 Ce qui donne avec la voyelle [a] tout en respectant le découpage rythmique :

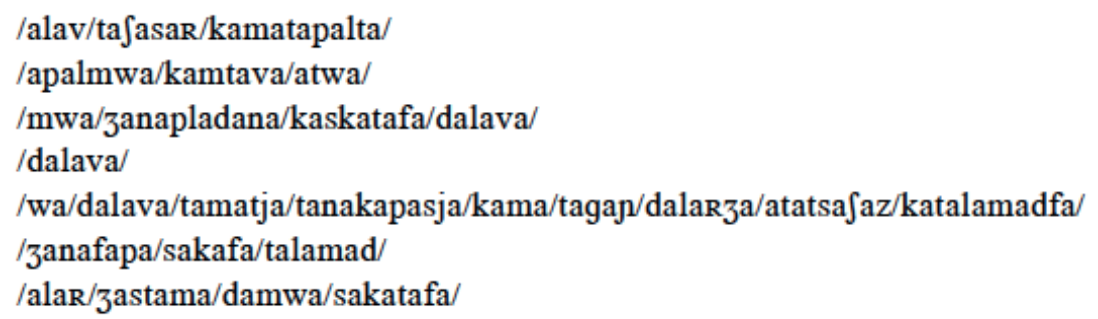

La deuxième étape consiste à ne prononcer que les voyelles, sans les enchaîner, afin de bien les différencier :

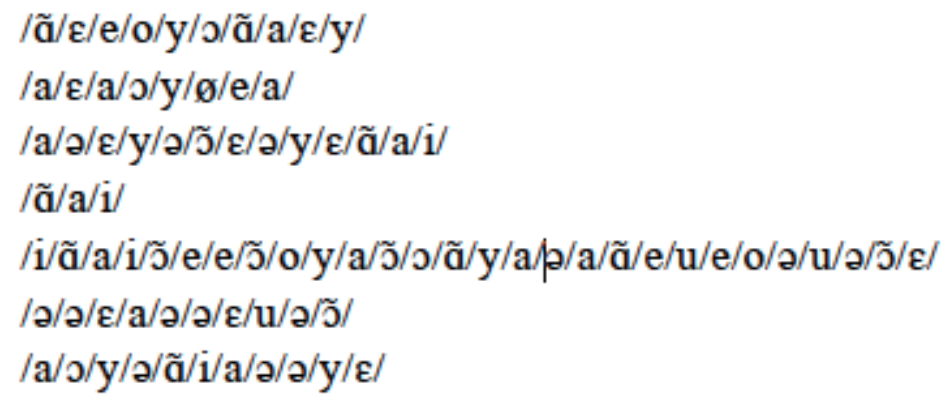

11 La troisième étape consiste à ne prononcer que les voyelles, en les enchaînant, pour retrouver une certaine fluidité, en respectant le découpage rythmique :

\section{/ã $\varepsilon /$ eoy/วãąy/ \\ /aєa/ıyø/ea/...}

La quatrième étape consiste à redire le texte, et à noter les améliorations. En plus de rendre la diction plus précise et plus limpide, cet exercice facilite aussi la mémorisation du texte, grâce à la mémoire kinesthésique stimulée par la gymnastique articulatoire. 
13 Avant la lecture, l'enseignant fera un travail sur le texte en délimitant les unités rythmiques, les syllabes, les liaisons, les enchaînements, les chutes de "e ", et les voyelles accentuées. L'apprenant prendra conscience des différences entre l'oral et l'écrit propres au français. Cependant, il sera toujours confronté à des phonèmes qui n'existent pas dans sa langue. Aussi est-il nécessaire au préalable, de l'amener à découvrir des lieux d'articulations différents, en prenant conscience des gestes articulatoires et du rôle des organes phonateurs.

\section{2. Explorer les mouvements des organes de la parole}

14 La volonté de bien articuler risque souvent de provoquer une diction artificielle. Un acteur cherchera plus à travailler la vélocité, et la fluidité de son élocution, afin de pouvoir varier son débit, d'éviter que le texte soit un obstacle à ses intentions de jeu. Rechercher cette fluidité, cette souplesse articulatoire peut être bénéfique à l'apprenant, car le fait de ne se concentrer que sur la bonne articulation des phonèmes peut nuire à la fluidité de la prosodie du français. Plutôt que de forcer l'articulation et de créer des tensions dans la mâchoire, il s'avère préférable d'explorer toutes les possibilités de mouvements articulatoires, et de les rendre plus souples.

exercices de diction les plus classiques consistent souvent à muscler les lèvres, les mâchoires et la langue, grâce à certaines contraintes musculaires, comme le fait de dire un texte avec un stylo, ou un bouchon placé entre les incisives, ou de faire prononcer des virelangues, véritables exercices d'habileté articulatoire. Ces derniers sont souvent utilisés en classe de langue étrangère. Ils séduisent par leur caractère ludique. Ils motivent les apprenants. Néanmoins, le défi, à savoir celui de prononcer les phrases sans se tromper, s'avère également difficile à relever même pour les natifs, pourtant habitués aux phonèmes à prononcer. Ces exercices font plus office d'entraînement, une fois que l'articulation des phonèmes est plus ou moins maîtrisée.

Citons quelques virelangues pour travailler :

- les voyelles nasales : un bon plein verre de bien bon vin blanc vieux.

- le [y] : À l'heure où le loup lut au lit, le hibou hulula à la nuit.

- le [s] et le []] : Sachez laisser sécher ses sachets.

17 Parmi les travaux de recherche pour acteurs, chanteurs et tout autre corps de métier utilisant la voix, figurent ceux de François Combeau (2001), orthophoniste, praticien et formateur de la méthode Feldenkrais ${ }^{\circledast}$, méthode d'apprentissage par le mouvement. Ses séances de travail, disponibles sur un $\mathrm{CD}$ intitulé Les outils d'une articulation claire et précise, proposent une véritable exploration en mouvement de tout l'appareil phonateur.

Plus connue chez les physiothérapeutes, la méthode Feldenkrais ${ }^{\circledast}$ (1977) invite à la prise de conscience de soi en mouvement. Il s'agit souvent de mouvements très doux, et très simples, que l'on répète jusqu'à ce qu'ils deviennent les plus fluides possible. L'attention est plus portée sur le processus et le ressenti du mouvement que sur le résultat et la réussite. Il s'agit plus d'explorer le mouvement que de reproduire approximativement une posture. Selon Combeau (2001), «dans ce travail [...] le mouvement de l'ensemble ou d'une partie est une occasion de recréer un vrai dialogue sensorimoteur, d'accorder action et sensation, de développer le sens kinesthésique et 
ses nombreux capteurs au niveau des différents systèmes osseux, articulaires, musculaires".

Adapter cette méthode peut se révéler utile en classe de langue. Amener les apprenants à découvrir ou redécouvrir leur propre appareil phonateur et sa motricité peut leur permettre de visualiser de manière plus concrète les différents lieux d'articulation, et d'assimiler les gestes de l'appareil phonateur. On se rapproche des principes de la méthode articulatoire, mais sans "avoir une connaissance explicite d'un son pour pouvoir bien le prononcer " (Billières et al., 2013). Plutôt que de "s'appuyer sur un apprentissage intellectualisé qui essaie de mettre en place les mouvements nécessaires à une bonne articulation » (Abry et Veldeman-Abry, $2007: 52$ ), la méthode Feldenkrais ${ }^{\circledast}$ invite à explorer un mouvement volontaire, et de le répéter, ce qui permet au système nerveux de l'intégrer dans son fonctionnement. De plus, ces exercices permettent à chaque organe de se mouvoir indépendamment des autres, ce qui aide à résoudre progressivement divers problèmes de coarticulation de phonèmes.

Avant de réaliser les exercices, il convient de conduire l'apprenant à se concentrer sur son appareil phonateur. Nous avons en effet tous une représentation peu précise de ce qui n'est pas visible. Pour la plupart d'entre nous, articuler se limite à un geste d'ouverture et de fermeture de la bouche, et les rôles de la langue, des lèvres et du voile du palais ne sont pas toujours clairs.

21 Afin d'avoir une image plus nette de la cavité buccale, il est possible de fermer les yeux, de prendre conscience des mouvements de la respiration, puis progressivement d'être attentif à la position des lèvres, d'observer si elles sont entrouvertes ou fermées, crispées ou détendues, puis de la langue, tout en sentant où est positionnée sa pointe, si elle est contre les dents ou plutôt en arrière, jusqu'à progressivement localiser la racine. On peut ensuite explorer toutes les zones du palais avec la pointe de la langue pour en sentir les différentes textures, et visualiser la forme de la voûte palatale et le volume de la cavité buccale. Il s'agit ensuite d'ouvrir et de fermer doucement la bouche, et de porter toute son attention aussi bien sur le mouvement d'ouverture que sur celui de fermeture comme si on le réalisait la première fois. Redécouvrir des gestes habituels et que l'on réalise inconsciemment facilite le processus d'exploration, et aide à ne pas se laisser piéger par ses propres mécanismes. Grâce à cette étape d'auto-observation, les apprenants non seulement se concentrent sur eux-mêmes physiquement, la classe étant souvent un lieu où ils font surtout appel à leurs capacités cognitives, mais aussi se tranquillisent. En effet, travailler sur les organes de la bouche peut être vécu comme une intrusion, et provoquer une certaine gêne.

Les erreurs phonologiques régulièrement produites par les apprenants de français langue étrangère sont dues dans un premier temps à une mauvaise perception, mais aussi à une absence de conscience des mouvements articulatoires, et ceci, même dans leur propre langue.

Afin de clarifier le rôle de la langue, il s'agit d'abord de ne pas la considérer comme un organe qui se contente de se lever, d'avancer ou de reculer dans son intégralité. La langue est un organe complexe, que l'on peut dissocier en trois zones : la pointe, la zone prédorsale et la zone dorsale. Un mouvement très simple consiste à poser la pointe de la langue contre l'arrière des incisives inférieures, et d'avancer lentement la zone prédorsale de la langue, puis de la faire revenir à sa position initiale, tout aussi lentement, sans compenser par un mouvement du menton, l'objectif étant de bien mobiliser cette zone de la langue, qui joue un rôle dans l'articulation des voyelles 


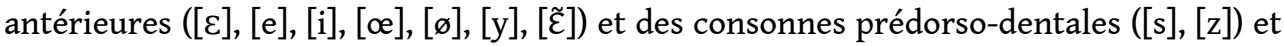
apico-palatales ([S], [3]). Pour rendre le mouvement plus libre, plus précis, et plus fluide, François Combeau propose dans un premier temps de lever les yeux quand la langue avance, et de regarder devant soi quand elle recule, plusieurs fois, jusqu'à ce que ce mouvement soit naturel. Pour approfondir l'exploration, on répétera le même mouvement, mais cette fois en baissant les yeux quand la langue avance. La dernière étape consistera à alterner le regard, vers le haut, puis vers le bas à chaque avancée de la zone avant de la langue ${ }^{2}$.

De la même manière, pour mieux arrondir et projeter les lèvres en avant, le mouvement consiste à dérouler la lèvre supérieure avec les doigts tout en levant, puis en baissant les yeux. Une seconde étape consiste à laisser les doigts sur la lèvre et baisser la tête, de façon à ce que l'inclinaison de la tête provoque le "retournement " de la lèvre ${ }^{3}$. Ce mouvement engendre une véritable prise de conscience du volume de la lèvre supérieure et de sa mobilité, ce qui facilite la protrusion nécessaire pour prononcer de façon plus claire les voyelles arrondies, et permet de différencier les consonnes labiales des labiodentales.

Pour travailler les mouvements du voile du palais, il s'agit davantage de faire appel à la capacité mentale de l'apprenant à imaginer un mouvement. On le conviera d'abord à parcourir les différentes zones du palais avec la pointe de la langue pour en apprécier les différentes textures, puis à se concentrer sur la zone molle du palais. On lui proposera ensuite d'imaginer que la voûte palatale descend puis monte vers la narine droite, l'œil droit, l'oreille droite, puis la même chose vers le côté gauche ${ }^{4}$.

Cette exploration mobile des organes de la parole permet donc d'assimiler des sons inhabituels, et d'acquérir une certaine fluidité, nécessaire également à l'acquisition de la prosodie du français. Chaque exercice dure environ une dizaine de minutes. Il est recommandé de les proposer séparément, et de les choisir selon le texte à interpréter. Le travail sur la zone prédorsale de la langue et sur l'arrondissement des lèvres s'avère pertinent pour préparer des textes dont l'auteur joue sur les voyelles arrondies, telle la chanson "Amoureuse " de Barbara, ou aussi, pour des apprenants d'un niveau plus avancé, sur les oppositions entre voyelles antérieures et postérieures, et sur les consonnes labiales et dentales, tels que des extraits de la première scène des Femmes savantes de Molière (1672, acte I, scène 1), où le personnage d'Henriette prononce des sons plus arrondis et plus graves, tandis qu'Armande prononce des sons plus antérieurs, plus tendus et plus aigus

Henriette : - Oui, mais tous ces soupirs chez vous sont choses vaines,

Et vous ne tombez point aux bassesses humaines

Votre esprit à l'hymen renonce pour toujours

Et la philosophie à toutes vos amours [...]

Armande :- Mais à l'offre des vœux d'un amant dépité

Trouvez-vous je vous prie entière sûreté ? [...]

Et croyez, quand il dit qu'il me quitte et vous aime

Qu'il n'y songe pas bien et se trompe lui-même.

Pour accentuer ce contraste entre les deux personnages, on peut varier les vitesses d'élocution comme le préconise la méthode verbotonale (Billières et al., 2013), et donc ralentir le débit pour les répliques d'Henriette et l'accélérer pour celles d'Armande.

Le travail sur le voile du palais s'adapte à des textes riches en voyelles nasales, comme « Le Chat » de Baudelaire (1857) : 
Dans ma cervelle se promène

Ainsi qu'en son appartement,

Un beau chat, fort, doux et charmant.

Quand il miaule, on l'entend à peine,

Tant son timbre est tendre et discret ;

Mais que sa voix s'apaise ou gronde,

Elle est toujours riche et profonde.

C'est là son charme et son secret. [...] que ses capacités cognitives. En répétant plusieurs fois le texte pour mieux le retenir, on lui impose inconsciemment un rythme et un phrasé, surtout si le texte est écrit en vers, ou si l'auteur crée des effets de styles. L'apprenant de langue étrangère risque souvent de contracter de mauvais automatismes lors de la mémorisation, tels que des accents mal placés, des erreurs phonologiques, ou des intonations fausses. Pour éviter d'enfermer le texte dans une même musique, l'acteur a recours à différentes techniques, reposant notamment sur un travail physique. Le corps et le geste ont une grande incidence sur la voix, la respiration, et donc les intonations. Selon Lauret (2007: 124), les « techniques [théâtrales] augmentent également l'assurance de l'élève, parce qu'elles l'aident à parler plus clairement, plus fort, et avec une variété de tons et de registres; le tout lié aux postures et mouvements du corps ».

\section{1. Faire corps avec le texte}

Il est fréquent, lorsque l'on récite un texte, qu'on ne sache pas quoi faire de ses bras, que l'on adopte une posture assez raide, et que l'on lève les yeux pour chercher dans sa mémoire. On observe également souvent, lors des répétitions ou dans les ateliers théâtres, que l'acteur ou l'élève oublie son texte quand il doit effectuer un déplacement, accomplir une action, ou tout simplement modifier sa posture corporelle. Ne plus dissocier le corps et la parole est une des bases fondamentales, du travail de l'acteur.

Tout comme pour les différentes intonations, il s'agit d'éviter d'adopter une gestuelle codée. Lors des répétitions, l'acteur explore donc plusieurs postures, plusieurs gestes, et reste attentif à la manière dont son corps influe sur sa voix, et son élocution. On offre 
rarement aux apprenants de langue étrangère l'occasion de se mouvoir, de modifier leur gestuelle. Or ceci permet d'éviter les automatismes. Travailler l'interprétation d'un texte tout en mobilisant son corps, permet d'explorer plusieurs intonations, lesquelles viennent naturellement du mouvement physique ou de la posture adoptée.

Le dramaturge et metteur en scène Augusto Boal (1994), dont l'essentiel du travail repose sur le corps, propose de nombreux jeux et exercices, permettant de rompre les mécanismes des gestes, des intonations, souvent dictés par le système cognitif, et de développer l'écoute, le toucher, et la conscience corporelle. Certains s'avèrent particulièrement intéressants à appliquer à un travail sur le texte, dans la mesure où ils invitent à adopter des postures inhabituelles, ou non volontaires :

- Hypnose colombienne. Un acteur met sa main à quelques centimètres du visage d'un autre qui est alors comme hypnotisé et doit constamment maintenir son visage à la même distance de la main de l'hypnotiseur : les cheveux et les doigts, le menton et le poignet. L'hypnotiseur commence une série de mouvements avec sa main, de haut en bas, à droite et à gauche, en avant et en arrière, la main verticale par rapport au sol, horizontale, en diagonale, son camarade doit faire toutes les contorsions possibles pour garder toujours la même distance (Boal, 1994 : 85).

- Le miroir simple. Deux files de participants, chacun regardant, les yeux dans les yeux, la personne qui lui fait face. Ceux de la file A sont les « sujets", ceux de la file B sont les «images». L'exercice commence. Chaque sujet entreprend une série de mouvements et d'expressions de physionomie, que son « image» doit copier dans les moindres détails [...] Au bout de quelques minutes le joker qui mène l'exercice, annonce que les deux files vont changer de rôles (ibid. : 136).

- Le sculpteur touche le modèle. Les participants se placent en deux files face à face. Une des files est composée de sculpteurs et l'autre de statues. Au début de l'exercice, chaque sculpteur commence à modeler de ses mains la statue qu'il imagine (ibid. : 142).

Il s'avère pertinent de les réaliser en classe, une fois le texte appris. Il est en effet concevable de prononcer le texte tout en les faisant. Ces différentes contraintes à l'interprétation permettent tout d'abord de rompre les rythmes et les mélodies que l'on aura pu figer en mémorisant le texte. Le fait de se retrouver dans des postures improvisées, de devoir faire des contorsions, ou de se retrouver en équilibre, oblige à modifier la respiration ainsi que le timbre de la voix. L'acteur, ou l'apprenant découvre alors toute une série de variations possibles. Lors de ces exercices, le corps guide progressivement la parole, effaçant ainsi toute dissociation, et permettant à celui qui les exécute de puiser plus en lui-même, ce qui l'aidera à se libérer peu à peu des contraintes de prononciation, et à aller au-delà de la simple imitation.

De plus, il s'agit également ici de développer l'écoute du partenaire, nécessaire à toute interaction. En effet, un acteur qui ne prend pas en compte son partenaire s'enferme très vite dans un jeu machinal. L'écoute, la prise de conscience de l'instant présent, sont des éléments essentiels à la disponibilité de l'acteur, laquelle est nécessaire pour adapter son jeu à la situation. La classe est également un espace d'interactions. Travailler l'écoute active, prendre conscience des pairs aide l'apprenant à aiguiser sa perception auditive, à réagir de manière plus spontanée, et à se libérer de certains mauvais mécanismes enkystés.

Après ces exercices, lors d'une nouvelle lecture à voix haute, l'extrait théâtral travaillé se retrouve généralement libéré d'effets parasites recherchés par ceux qui le prononcent. Les phrases sont plus distinctes, les intonations plus naturelles, les silences plus présents. 


\section{2. Travailler la spontanéité pour trouver le ton juste}

L'exercice de « la répétition », créé par Sanford Meisner (Meisner et Longwell, 1987), se révèle très utile pour libérer les tensions des apprenants, et surtout pour qu'ils restent très conscients de l'instant présent, et soient totalement connectés à leur interlocuteur.

Cet exercice se déroule en plusieurs étapes. Il consiste à mettre deux acteurs, A et B, face à face. A dit quelque chose de réel à $B$. Il doit s'agir d'un constat et non d'un jugement. B répète la phrase de A. La même phrase doit être répétée plusieurs fois pour laisser surgir plusieurs intonations.

Exemple :

A : tu as un pull bleu

$B$ : tu as un pull bleu.

A : tu as un pull bleu

$\mathrm{B}:$ tu as un pull bleu.

etc.

L'exercice peut évoluer de la façon suivante :

$A:$ tu as un pull bleu

$B$ : j'ai un pull bleu.

etc.

Il convient de bien observer son partenaire pendant tout l'exercice, et d'être à l'écoute de tous ses propres changements d'état. Il est également intéressant de faire évoluer l'exercice et de demander aux acteurs de changer de phrase dès qu'ils observent un changement, l'objectif étant d'être totalement conscient de l'instant présent.
A : tu as un pull bleu
$B$ : j'ai un pull bleu.
A : tu as un pull bleu
$B$ : j'ai un pull bleu.
A : tu tournes la tête
B : je tourne la tête.
A : tu tournes la tête
B : je tourne la tête.
A : tu tournes la tête
$B$ : tu souris
A : je souris...

On peut tout à fait adapter cet exercice favorisant l'improvisation à un travail sur un texte de théâtre contemporain, aux répliques courtes, comme des extraits de pièce d'Israël Horovitz, Michel Azama, Catherine Anne, pour ne citer qu'eux. Il suffit de commencer l'exercice, et quand le meneur de jeu tape dans ses mains, dire le texte en tentant de garder la même qualité d'écoute et d'observation. Dès qu'il sent que les acteurs ou élèves s'installent dans une musique mécanique, le meneur de jeu retape dans ses mains et les acteurs ou élèves passent de nouveau à l'exercice de la répétition. Le fait de porter toute son attention sur son partenaire et non sur soi-même rend beaucoup plus présent, et spontané. Lors de l'exercice, les intonations varient naturellement, sans qu'elles soient préméditées.

Rompre les mécanismes ne consiste pas seulement à éviter de reproduire des erreurs. Il s'agit aussi de lâcher prise, de développer l'écoute, d'aiguiser son sens de l'observation. Après ces exercices, les apprenants de langue étrangère pourront reproduire quelques 
erreurs. Mais ils seront beaucoup plus disponibles, ce qui facilitera la correction des erreurs, et leur prise de conscience de ces dernières.

\section{Conclusion}

Travailler l'interprétation du texte théâtral en classe de langue, qu'il soit classique ou contemporain, permet d'aller au-delà du simple entraînement phonétique. Il s'agit d'être capable non seulement d'articuler correctement les phonèmes, mais aussi de varier les rythmes, les intonations, tout en respectant le sens, et en appréciant la musicalité recherchée par l'auteur.

L'objectif n'est pas de faire des apprenants des acteurs professionnels, mais bien de leur permettre de s'ouvrir, tout en découvrant organiquement un texte d'auteur, à un véritable travail d'exploration et d'auto-observation, de développer leur capacité d'écoute, de les inviter à lâcher-prise pour les aider à mieux s'approprier physiquement la langue, ses sons et son phrasé, afin de pouvoir exprimer progressivement des nuances de sens, et d'interagir plus librement, lors de la prise de parole spontanée.

\section{BIBLIOGRAPHIE}

ABRY D. et VELDEMAN-ABRY J., 2007, La phonétique : audition, prononciation, correction, Paris, CLE International, coll. « Techniques et pratiques de classe ».

ABRY D. et CHALARON M.-L., 2010, Les 500 exercices de phonétique : niveau A1-A2, Paris, Hachette, coll.

«étranger».

-, 2011, Les 500 exercices de phonétique : niveau B1-B2, Paris, Hachette, coll. « étranger ».

BILLIÈRES M. et al., 2013, Phonétique corrective en FLE. Méthode verbo-tonale. Disponible sur : <http://

w3.uohprod.univ-tlse2.fr/UOH-PHONETIQUE-FLE/index.html>.

BOAL A., 1994 [1989], Jeux pour acteurs et non-acteurs. Pratique du théâtre de l'opprimé, Paris, La

Découverte, coll. « Malgré tout».

COMBEAU F., 2001, «Interview de François Combeau. Présentation de la Méthode Feldenkrais ${ }^{\circledR}$ dans

le cadre d'un colloque sur “Moyens d'investigation et pédagogie de la voix chantée”, organisé à

Lyon (10 février 2001) », Espace du temps présent. Disponible sur : <http://training-feldenkrais-

francois-combeau.com/articles/french/interview-de-francois-combeau>.

FELDENKRAIS M., 1977, Awareness through movement : easy-to-do health. Exercises to improve your posture, vision imagination, and personal awareness, New York, Harper et Row.

KOLTÈS B.-M., 1990, Roberto Zucco, Paris, Éditions de Minuit.

LAURET B., 2007, Enseigner la prononciation du français : questions et outils, Paris, Hachette, coll. «f ».

LIARD M., 2007, Parole écrite, parole scénique (du texte à la scène), Nantes, Joca Seria. 
MEISNER S. et LONGWELL D., 1987, Sanford Meisner on acting, New York, Vintage books.

RENUCCI R. (avec Isabelle Francq), 2014, Tous ces hasards qui n'en sont pas, Paris, Presses de la Renaissance, coll. «Chemin faisant ».

\section{Sitographie}

BILLIÈRES M., Au son du fle. Disponible sur : <https://www.verbotonale-phonetique.com/

phonetique/>.

Formations Feldenkrais. Disponible sur : <http://formations-feldenkrais-francois-combeau.fr/>.

\section{NOTES}

1. Cet exercice s'avérant assez difficile et nécessitant une certaine connaissance des différentes graphies, du découpage rythmique, des phénomènes de liaisons, d'enchaînement, s'applique plutôt à des apprenants ayant déjà acquis un niveau intermédiaire.

2. Développer les mobilités différenciées langue mâchoires : séance dite de la langue.

3. Le retournement des lèvres et la mobilité de la tête, des yeux et du bassin.

4. Découvrir, localiser, mobiliser le voile du palais : développer la conscience de l'espace.

\section{RÉSUMÉS}

Interpréter un texte figure parmi les pratiques de classe de langue étrangère. Néanmoins cette activité se limite souvent à la simple mémorisation et répétition de phrases et de schémas intonatifs, ce qui peut générer certains mécanismes, empêchant alors l'apprenant de pouvoir faire sienne la parole en langue étrangère, et de se rendre plus disponible afin de se corriger plus facilement. Certaines techniques d'acteur méconnues par les enseignants, car peu diffusées, s'avèrent très utiles pour obtenir une articulation précise et fluide, varier les intonations, et s'approprier le texte, et ainsi adopter progressivement les sons, le rythme et les mélodies de la langue cible.

Interpreting a dialog or a text is one of the practices in foreign language learning classes but it is often limited to mere repetition and memorization of sentences and intonation schemes which may result in a mechanical approach to the foreign language instead of a more personal approach which would allow more flexibility and self-correction capacity. That is why some acting techniques which teachers may not be aware of for lack of information can prove very useful to improve precision and fluidity in articulation and more variety in intonations, and therefore gradually adopting sounds, rhythm and melodies of the target language. 
INDEX

Mots-clés : techniques d'acteur, interpréter, articulation, fluidité, spontanéité, disponibilité, méthode Feldenkrais ${ }^{\circledast}$, méthode Meisner.

Keywords : Acting technique, interpreting, articulation, fluidity, spontaneity, availability, Feldenkrais ${ }^{\oplus}$ method, Meisner method

\section{AUTEUR}

\section{FRANÇOIS BLONDEL}

Institut français de Madrid 